revision surgery. 146 had histologically confirmed thyroglossal duct cysts. Alternative pathologies were: 16 dermoid cysts, 2 ectopic thyroid tissue foci, 1 scar tissue only, 1 mucus retention pseudocyst, 1 development cyst \& 1 fourth arch anomaly.

26 patients experienced a minor post-operative complication (managed conservatively). 4 patients required further operative intervention: 2 revision surgery and 2 drainage of haematoma. Overall recurrence rate was 1 (1.6\%).

Conclusion We present the largest published case series of patients who have undergone the extended Sistrunk's procedure. We accept the small increase in conservatively managed wound complications (e.g. wound swelling or minor haematomas) and note the very low return to theatre rate. We advocate for use of this technique even for primary cases as the recurrence rate is low with little peri- or post-operative morbidity.

\section{INVESTIGATING THE MODIFIABLE PSYCHOSOCIAL VARIABLES INFLUENCING ACCESS TO AND OUTCOMES AFTER KIDNEY TRANSPLANTATION IN CHILDREN - A STUDY PROTOCOL}

${ }^{1} \mathrm{Ji}$ Soo Kim, ${ }^{1}$ Jo Wray, ${ }^{2}$ Stephen Marks. ${ }^{1}$ Great Ormond Street Hospital for Children NHS Foundation Trust; ${ }^{2}$ Paediatric Nephrology, Great Ormond Street Hospital for Children NHS Foundation Trust

\subsection{6/archdischild-2020-gosh.108}

Introduction Kidney transplantation, compared with dialysis, is often seen as the gold standard in optimising health, reducing mortality and improving quality of life in children with End Stage Kidney Disease (ESKD). We recently surveyed 12 out of 13 UK paediatric nephrology centres on their transplantation plans for all children registered with ESKD. The most commonly cited factors delaying kidney transplantation in these children were: disease-related (36\%), availability of a suitable donor (27\%) and the child's size (20\%). In 19\% of children, psychosocial factors were listed as a barrier. Some factors, including psychosocial, may be modifiable through local or national intervention. To inform future interventions, further study is needed to explore the range and nature of these psychosocial factors.

Aim To investigate the psychosocial factors that influence access to and outcomes of kidney transplantation among children in the UK.

Methods and Analysis This is a prospective multicentre (13 UK paediatric nephrology centres) mixed-methods study with QUAL-QUANT and QUANT-QUAL phases. First, we will use thematic analysis to review interviews conducted with NHS professionals, children with ESKD and their families that explore these psychosocial factors. Next, validated questionnaires that measure these psychosocial factors will be distributed to the wider UK cohort of pre-transplant children with ESKD and their families. They will be followed up to 2 years regardless of whether they do or do not receive a kidney transplant. Clinical data will be prospectively collected from local hospital notes and registry data (UK Renal Registry and NHS Blood \& Transplant). Families with outlier results will be invited for further interview to explore their findings.

\section{9 COCATOO: A COHORT COMPARISON TOOL FOR GOSH CLINICAL RESEARCHERS}

${ }^{1}$ Lydia Briggs, 'William Bryant, 'Mohsin Shah, 'John Booth, 'Richard Issitt, ${ }^{1}$ Anastassia Spiridou, ${ }^{2}$ Neil I Sebire. ${ }^{1} D R I V E$, Great Ormond Street Hospital for Children NHS Foundation Trust; ${ }^{2}$ GOSH DRIVE

10.1136/archdischild-2020-gosh.109

When faced with large amounts of data, it can be a challenging and lengthy process for a researcher to identify key differences and trends between different sets of patients. The development of a reporting tool which can be easily adapted to any speciality and patient selection would be advantageous in highlighting and presenting patient group comparisons in a clinical research environment.

By developing standard, reproducible analytics from the core datasets provided by the Digital Research Environment (DRE) team at GOSH, the researcher is able to identify differences in hospital (hospital admissions, ward stays) and clinical (patient laboratory results, demographics and vital signs) trends between user defined cohort-control patient groups which can accelerate their clinical understanding.

Herein, we introduce COCATOO, a cohort comparison tool that compares and reports distinct sets of patient groups based on defined specifications which are controlled by the researcher. By utilising generalisable analytics developed in $\mathrm{R}$, the tool is able to display a set of predefined exploratory data analytics (EDA) which have the ability to be adapted to any hospital department by incorporating project specific rules.

We present here an example with Chronic Kidney Disease (CKD) comparing stage 5 to stages $1-4$. The output report gives an overview of the disease and highlights significant cohort-control differences via the predefined EDA.

\section{CLINICAL AND OPERATIONAL INSIGHTS: WORKING TO EMBED DATA-DRIVEN PROCESSES AT GOSH}

${ }^{1}$ William Bryant, ${ }^{1}$ Lydia Briggs, ${ }^{1}$ Richard Issitt, ${ }^{1}$ Mohsin Shah, ${ }^{1} J o h n$ Booth, ${ }^{1}$ Anastassia Spiridou, ${ }^{2}$ Neil J Sebire. ${ }^{1} D R I V E$, Great Ormond Street Hospital for Children NHS Foundation Trust; ${ }^{2}$ GOSH DRIVE

\subsection{6/archdischild-2020-gosh.110}

With clinical and operational data for research more easily accessible than ever before through the Digital Research Environment (DRE) in DRIVE, it now is possible to use dataintensive methods and advanced analytics to address many challenges and provide new insights, both clinical and operational. Opportunities for innovation are plentiful and come in many forms, from dashboarding key indicators and trends, to predicting patient needs, forecasting service demand and more. With the expertise and experience gathered within the DRE Team, we are able to leverage key commonalities between projects and focus on particular challenges faced by diverse teams across the organisation.

Herein we introduce our workflow for data extraction, transformation, presentation and analysis and how we can use these to add value for both clinical and operational data use. We also discuss how we can translate requirements and constraints from various teams in order to drive refinements in data presentation and process optimisation. We present two 